\title{
Transcatheter Closure of Ventricular Septal Rupture First in Bangladesh - A Case Report
}

\author{
NAM Momenuzzaman, Solaiman Hossain, Fatema Begum, Kaisar Nasrullah Khan, \\ AM Shafique, KM Sohail \\ United Hospital Limited, Dhaka, Bangladesh
}

Key Words :
Ventricular septal
rupture, Acute
Myocardial
infarction,
Amplatzer device.

Abstract:

Ventricular septal rupture is a rare complication of acute myocardial infarction with important hemodynamic consequences. Without a rapid diagnosis and correction by surgical intervention, the short-term mortality of these patients is higher than 90\%. Early diagnosis is based on clinical examination and transthoracic echocardiography. Transcatheter closure of ventricular septal rupture in selected patients may save lives. We report a patient with ventricular septal rupture in acute myocardial infarction that was closed by an Amplatzer device.

(Cardiovasc. j. 2015; 7(2): 150-152)

\section{Introduction:}

Ventricular Septal Rupture (VSR) after acute myocardial infarction (AMI) is a fatal mechanical complication. VSR tends to occur 3 to 8 days after the AMI but may also occur within the first 24 hours, or as late as 2 weeks. ${ }^{1}$ In the era before thrombolysis, VSR was not an infrequent complication of AMI, occurring in as many as $1 \%$ to $3 \%$ of patients. ${ }^{1,2}$ With the advent of early reperfusion strategies and adjunct medical therapy, the incidence of this complication has significantly decreased to $<1 \%$ of cases $(0.2 \%$ in the Global Utilization of Streptokinase and Tissue Plasminogen Activator for Occluded Coronary Arteries-I [GUSTO-I] trial) but remains associated with a high morbidity and mortality. ${ }^{3}$

Development of a VSR correlates with delayed hospital admission after the infarction (> 24 hours) and may be triggered by undue physical activity in the early post infarction period, as well as recurrent ischemia. Risk factors of VSR include age $>60$ years, female gender, no history of previous MI and hypertension. ${ }^{4}$ The defect is most often at the apical septum in anterior AMI and the base of the heart after inferior AMI, with the orifice varying from 1 to several centimeters in diameter. ${ }^{5}$

Early surgical repair has been the only accepted treatment, but it carries very high perioperative mortality and is therefore applied only to selected patients. ${ }^{6}$ In recent years, percutaneous closure of postinfarction VSR has been proposed as an alternative to surgical repair and even to the conservative approach. However, experience in percutaneous closure of postinfarction VSR is very limited and restricted to selected patients. ${ }^{7,8}$ The patients treated without surgery mortality is about $90 \%$. Surgical treatment must be carried out on an emergency basis, even if the patient is stable. All VSRs are closed with a patch and associated coronary artery bypass grafting (CABG) operative mortality is $10-15 \%$ for anterior defects and 30 $35 \%$ for posterior defects. ${ }^{9}$

We report the case of a 43 years female with postinfarction VSR who was not considered suitable for surgical repair and thus it was treated with percutaneous closure using an Amplatzer device.

\section{Case Summary:}

This is the first case of Transcatheter closure of post infarction VSR in Bangladesh using ASD Amplatzer device. A 43 years normotensive diabetic lady admitted in our Hospital with the history of shortness of breath for 07 days. She was diagnosed as a case of anterior AMI with acute left ventricular failure with cardiogenic shock and was treated with Inj. Streptokinase in another cardiac center.

On admission, her Pulse- 118/min, BP- 90/50 $\mathrm{mmHg}$, a systolic murmur at left lower parasternal region, Lungs- Killip class III crepitation. Transthoracic Echocardiography revealed: severely hypokinetic mid-distal interventricular septum,

Address of Correspondence: Dr. Solaiman Hossain, Department of Cardiology, United Hospital LTD, Dhaka, Bangladesh. E-mail: hossainsolaiman@gmail.com 


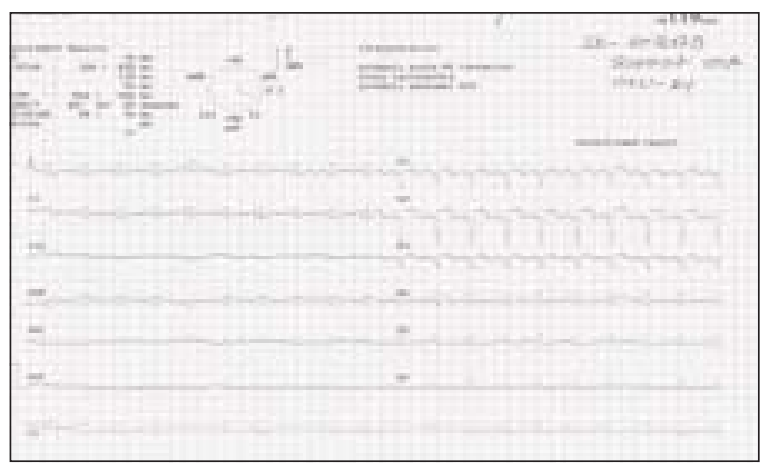

Fig.-1: ECG on admission.

apex, mid-distal anterior and anteroseptal walls, and moderate LV systolic dysfunction with LVEF: $42 \%$ and an echo-dropout about $10 \mathrm{~mm}$ seen at distal part of mid antero-septum consistent with VSR.

After admission, she was managed conservatively in CCU. After stabilization, CAG was done which revealed non critical coronary artery disease and Left Ventriculography revealed VSR. Our recommendation was Medical management of IHD and transcatheter closure of VSR.

\section{Technique:}

At the beginning of the procedure, the patient was put on mechanical ventilation. The procedure was performed under transesophageal echocardiography guidance. The right femoral artery and right femoral vein were catheterized. An extra stiff Amplatzer wire 0.35 X260 wire was passed through the right femoral artery and inserted at the left ventricle. It was advanced through the VSR to the right ventricle, right atrium and right femoral vein taken by a snare. A $12 \mathrm{~mm}$ Amplatzer device was advanced through the wire to the right ventricle and then to the left ventricle. The left side of the device was deployed first at the left ventricle, then the right side at the right ventricle. After confirming its optimal position in the VSR, the device was finally deployed. Left Ventriculography showed a mild persistent left to- right shunt.

The patient's hemodynamics improved and remained stable 24 hours after the procedure then extubated from mechanical ventilation. Transthoracic echocardiography performed the following day showed the Amplatzer device correctly positioned across the VSR and a minor residual shunt. After 03 days the patient is discharged with stable hemodynamic condition. The patient is on regular follow-up with complete closure of the residual shunt and normal daily activities.

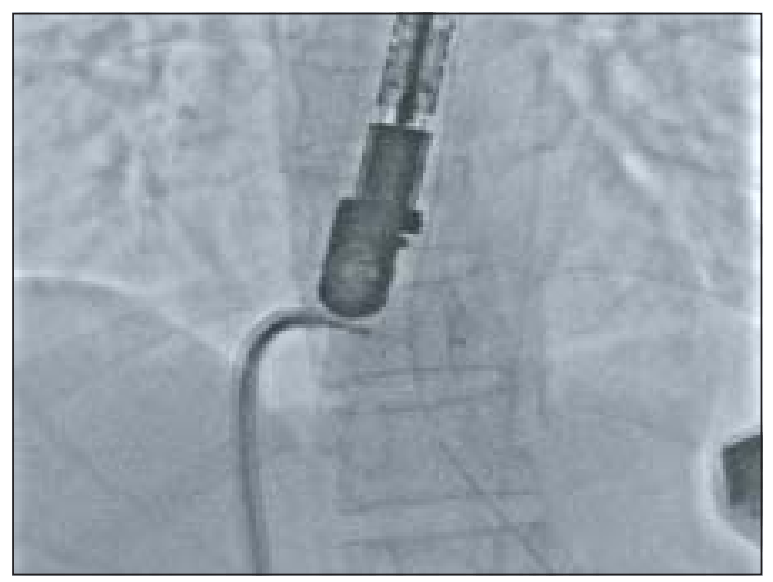

Fig.-2: Transcatheter deployment of Amplatzer Device in VSR under guidance of TEE.

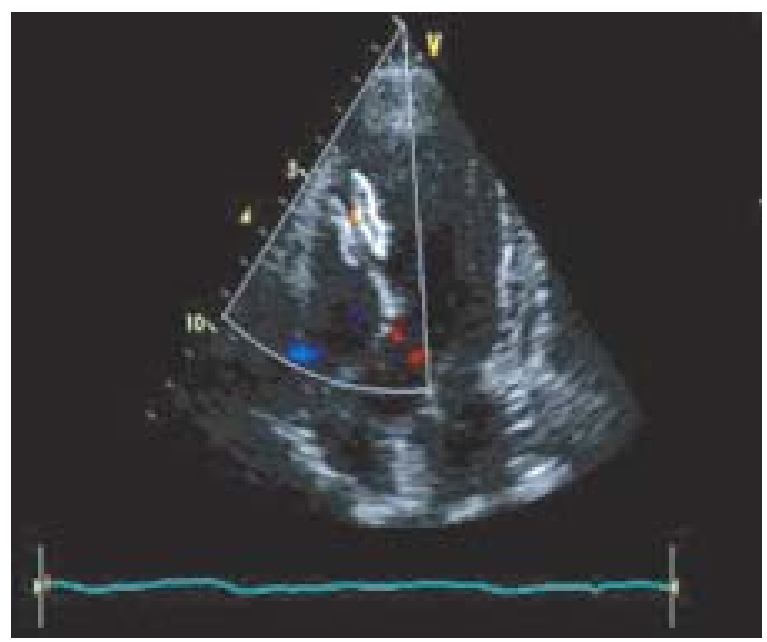

Fig.-3: TTE after deployment of device showing no residual shunt.

\section{Conclusion:}

Transcatheter device closure is an accepted alternative to surgery for the post-AMI VSR is expanding. Although cardiac surgery with concurrent coronary artery bypass grafting, as indicated, remains the gold standard, a significant proportion of patients are at too high risk for surgery. Transcatheter device closure may provide suitable procedure for noncritical coronary artery disease with suitable lesion. Patients with large 
or multiple defects are best treated surgically. A multidisciplinary team, including interventional cardiologists and cardiac surgeons, should evaluate these high-risk patients to determine the optimal management strategy and reduce high morbidity and mortality.

\section{Conflict of Interest - None.}

\section{References:}

1. Topaz O, Taylor AL. Interventricular septal rupture complicating acute myocardial infarction: from pathophysiologic features to the role of invasive and noninvasive diagnostic modalities in current management. Am J Med 1992; 93:683-688.

2. Heitmiller R, Jacobs ML, Daggett WM. Surgical management of postinfarction ventricular septal rupture. Ann Thorac Surg 1986;41:683-691.

3. Crenshaw BS, Granger CB, Birnbaum Y, et al. Risk factors, angiographic patterns, and outcomes in patients with ventricular septal defect complicating acute myocardial infarction. GUSTO-I (Global Utilization of Streptokinase and TPA for Occluded Coronary Arteries) Trial Investigators. Circulation 2000; 101:27-32.
4. Figueras J, Cortadellas J, Calvo F, et al. Relevance of delayed hospital admission on development of cardiac rupture during acute myocardial infarction: study in 225 patients with free wall, septal or papillary muscle rupture. J Am Coll Cardiol 1998; 32:135-139.

5. Edwards BS, Edwards WD, Edwards JE. Ventricular septal rupture complicating acute myocardial infarction: identification of simple and complex types in 53 autopsied hearts. Am J Cardiol 1984; 54:1201-1205.

6. Holzer R, Balzer D, Amin Z, et al. Transcatheter closure of postinfarction ventricular septal defects using the new Amplatzer Muscular VSD occluder: Results of a U.S. Registry. Catheter Cardiovasc Interv 2004; 61: 196-201.

7. Hachida M, Nakano H, Hirai M, et al. Percutaneous transaortic closure of postinfarctional ventricular septal rupture. Ann Thorac Surg 1991; 51: 655-657.

8. Szkutnik M, Bialkowski J, Kusa J, et al. Postinfarction ventricular septal defect closure with Amplatzer occluders. Eur J Cardio-Thorac 2003; 23: 323-327.

9. Gaudiani VA, Miller DG, Stinson EB, et al. Postinfarction ventricular septal defect: an argument for earlyoperation. Surgery Jan 1981; 89(1):48-55. 